CASE REPORT

\title{
Three-dimensional Ultrasound Diagnosis of Circumvallate Placenta Early in Second Trimester of Pregnancy
}

Riko Takayoshi ${ }^{1}$, Toshiyuki Hata ${ }^{2}$, Aya Koyanagi ${ }^{3}$, Ai Sakamoto ${ }^{4}$, Nobutsugu Oguni ${ }^{5}$, Tadashi Hashimoto ${ }^{6}$, Tomoyoshi Takata ${ }^{7}$, Takahito Miyake ${ }^{8}$

\begin{abstract}
We present three patients with a circumvallate placenta diagnosed by two-dimensional (2D)/three-dimensional (3D) ultrasound and HDlive early in the second trimester of pregnancy. The characteristic 2D sonographic findings were a thick placenta with its edges protruding into the uterine cavity. The unique 3D ultrasound characteristic of a circumvallate placenta early in the second trimester is a pizza-like appearance. Moreover, 3D ultrasound and HDlive can clearly differentiate partial from complete circumvallate placentas. Three-dimensional ultrasound and HDlive provide additional, useful information for the early second-trimester diagnosis of a circumvallate placenta.
\end{abstract}

Keywords: Circumvallate placenta, Early second trimester, HDlive, Prenatal diagnosis, Three-dimensional ultrasound.

Donald School Journal of Ultrasound in Obstetrics and Gynecology (2020): 10.5005/jp-journals-10009-1630

\section{INTRODUCTION}

The estimated prevalence of a circumvallate placenta is approximately $5-8 \% .{ }^{1}$ A circumvallate placenta is included in the placenta extrachorialis category, as the chorionic plate is smaller than the basal plate, with resulting marginal placental infarction, hemorrhage, fibrin deposition, and tethering of membranes. ${ }^{2}$ It is characterized by the inward insertion of chorioamniotic membranes from the edges toward the center of the placenta, leading to back folding of the placenta and fetal membranes toward the chorionic surface. ${ }^{1}$ Complications such as placental abruption, preterm labor, oligohydramnios, and intrauterine fetal death have been reported with a circumvallate placenta. ${ }^{3}$ There have been only four studies on three-dimensional (3D) ultrasound and HDlive diagnosis of a circumvallate placenta. ${ }^{4-7}$ However, there have been only two cases of a circumvallate placenta diagnosed with these techniques early in the second trimester of pregnancy. ${ }^{5,7}$ In the current study, we presented three additional patients diagnosed by 3D ultrasound and HDlive early in the second trimester of pregnancy.

\section{Case Description}

\section{CASe 1}

A 34-year-old pregnant Japanese woman, gravida 2, para 0, was referred to our outpatient department at 18 weeks and 1 day of gestation for routine obstetric examination. Two-dimensional (2D) sonography (Voluson E10, GE Healthcare Japan, Tokyo, Japan) revealed an increased thickness $(51.2 \mathrm{~mm}$ ) with peripheral, irregular edges on the fetal surface (Fig. 1). Central placental depression with everted placental edges and thick, rolled up placental margins resembling pizza edges surrounding the depressed placental center could be easily depicted using HDlive (Fig. 2). Moreover, a partial defect of everted placental edges was noted.

At 39 weeks and 6 days of gestation, an emergency cesarean section was performed due to a nonreassuring fetal heart rate pattern, resulting in a single male newborn weighing 3,600 g, with a length of $52 \mathrm{~cm}$. The umbilical artery $\mathrm{pH}$ was 7.34. The Apgar score was $8 / 9$ at 1 and 5 minutes, respectively. Placental examination
1,3,4,6,7 Department of Obstetrics and Gynecology, Miyake Clinic, Minami-ku, Okayama, Japan

${ }^{2,8}$ Department of Obstetrics and Gynecology, Miyake Clinic, Minamiku, Okayama, Japan; Department of Perinatology and Gynecology, Kagawa University Graduate School of Medicine, Miki, Kagawa, Japan ${ }^{5}$ Department of Obstetrics and Gynecology, Miyake Clinic Toiyacho Terrace, Kita-ku, Okayama, Japan

Corresponding Author: Toshiyuki Hata, Department of Obstetrics and Gynecology, Miyake Clinic, Minami-ku, Okayama, Japan; Department of Perinatology and Gynecology, Kagawa University Graduate School of Medicine, Miki, Kagawa, Japan, Phone: +81-(0)87-891-2174, e-mail: toshi28@med.kagawa-u.ac.jp

How to cite this article: Takayoshi R, Hata T, Koyanagi A, et al. Threedimensional Ultrasound Diagnosis of Circumvallate Placenta Early in Second Trimester of Pregnancy. Donald School J Ultrasound Obstet Gynecol 2020;14(2):157-160.

Source of support: Nil

Conflict of interest: None

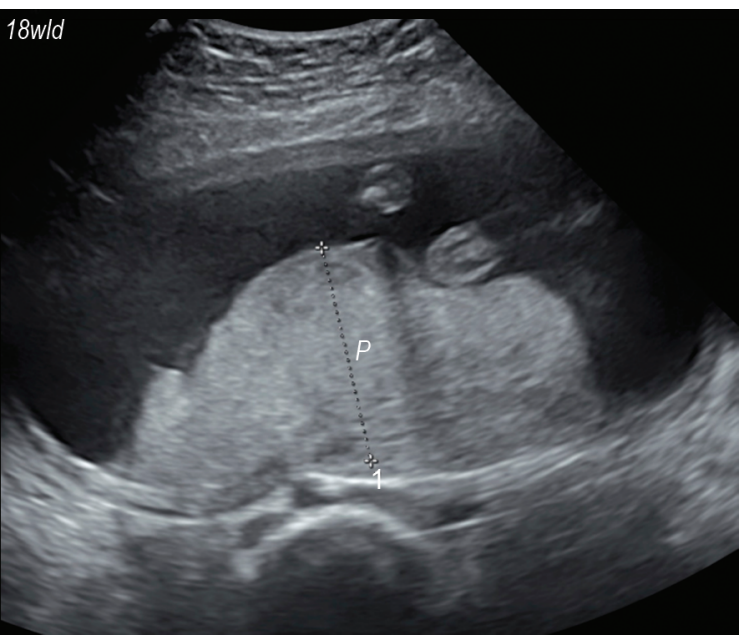

Fig. 1: A circumvallate placenta at 18 weeks and 1 day of gestation. Two-dimensional sonography shows placental edges protruding into the uterine cavity. Placental thickness is $51.2 \mathrm{~mm}$. P, placenta 


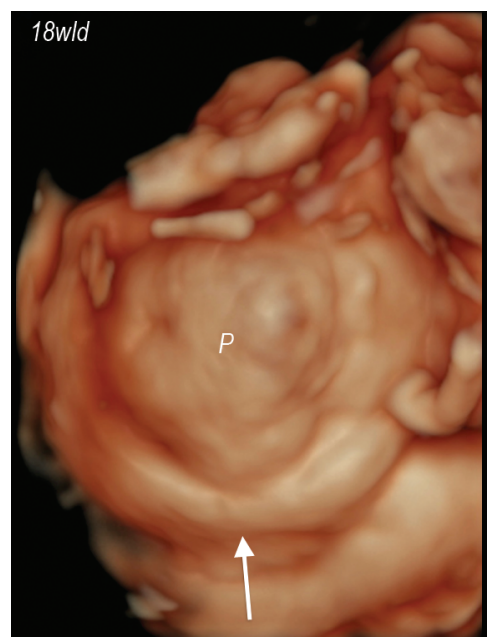

Fig. 2: A circumvallate placenta (arrow) at 18 weeks and 1 day of gestation. Central placental depression with everted placental edges and thick, rolled up placental margins resembling pizza edges surrounding the depressed placental center can be easily depicted using HDlive. Moreover, a partial defect of everted placental edges could be noted. P, placenta

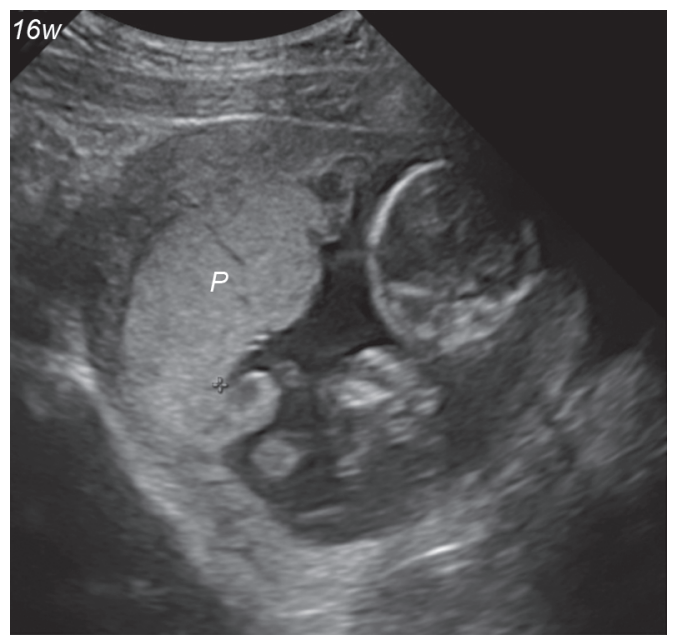

Fig. 4: A circumvallate placenta at 16 weeks of gestation. Twodimensional sonography shows placental edges protruding into the uterine cavity. Placental thickness is $33.3 \mathrm{~mm}$. P, placenta

revealed its weight to be $673 \mathrm{~g}$, and it showed a partial circumvallate placenta (Fig. 3).

\section{Case 2}

A 19-year-old pregnant Japanese woman, gravida 3, para 1, was referred to our outpatient department at 16 weeks of gestation for routine obstetric examination. Two-dimensional sonography revealed placental edges protruding into the uterine cavity (Fig. 4). The placental thickness was $33.3 \mathrm{~mm}$. Three-dimensional ultrasound showed a central placental depression with partially everted placental edges (Fig. 5).

One male newborn was vaginally delivered at 38 weeks and 6 days of gestation with a body weight of 3,104 $\mathrm{g}$ and a length of $50.5 \mathrm{~cm}$. The umbilical artery $\mathrm{pH}$ was 7.48. He had an Apgar score of $9 / 10$ at 1 and 5 minutes, respectively. Placental examination

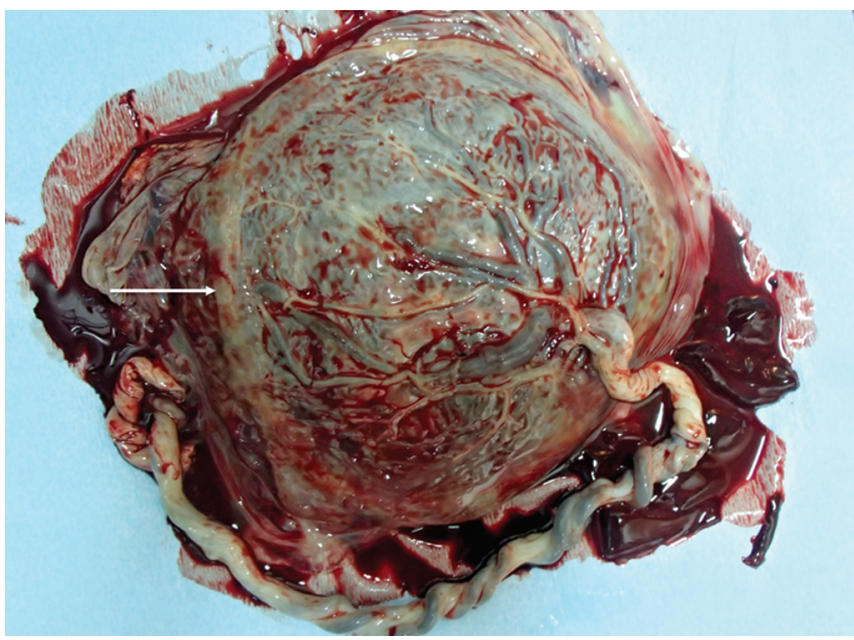

Fig. 3: Gross examination of a partial circumvallate placenta (arrow) after delivery

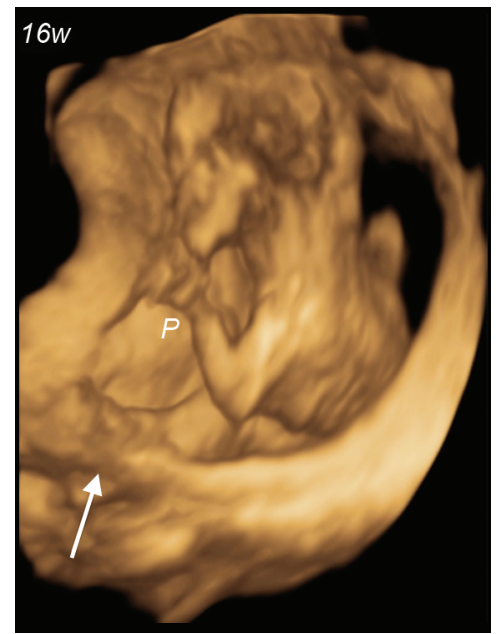

Fig. 5: A circumvallate placenta at 16 weeks of gestation. Threedimensional ultrasound shows a central placental depression with partial everted placental edges (arrow). P, placenta

revealed its weight to be $633 \mathrm{~g}$, and it showed a partial circumvallate placenta (Fig. 6).

\section{Case 3}

A 34-year-old pregnant Japanese woman, gravida 1, para 0, was referred to our outpatient department at 16 weeks and 1 day of gestation for routine obstetric examination. Two-dimensional sonography revealed placental edges protruding into the uterine cavity (Fig. 7). The placental thickness was $36.5 \mathrm{~mm}$. Threedimensional ultrasound showed a central placental depression with everted placental edges and thick, rolled up placental margins resembling pizza edges surrounding the depressed placental center could be easily depicted (Fig. 8).

At 38 weeks and 1 day of gestation, the elective cesarean section was performed due to a low-lying placenta, resulting in a single female newborn weighing $2,438 \mathrm{~g}$, with a length of $46 \mathrm{~cm}$. The umbilical artery $\mathrm{pH}$ was 7.39. The Apgar score was $9 / 10$ at 1 and 5 minutes, respectively. Placental examination revealed its weight to be $658 \mathrm{~g}$, and it showed a typical circumvallate placenta (Fig. 9). 


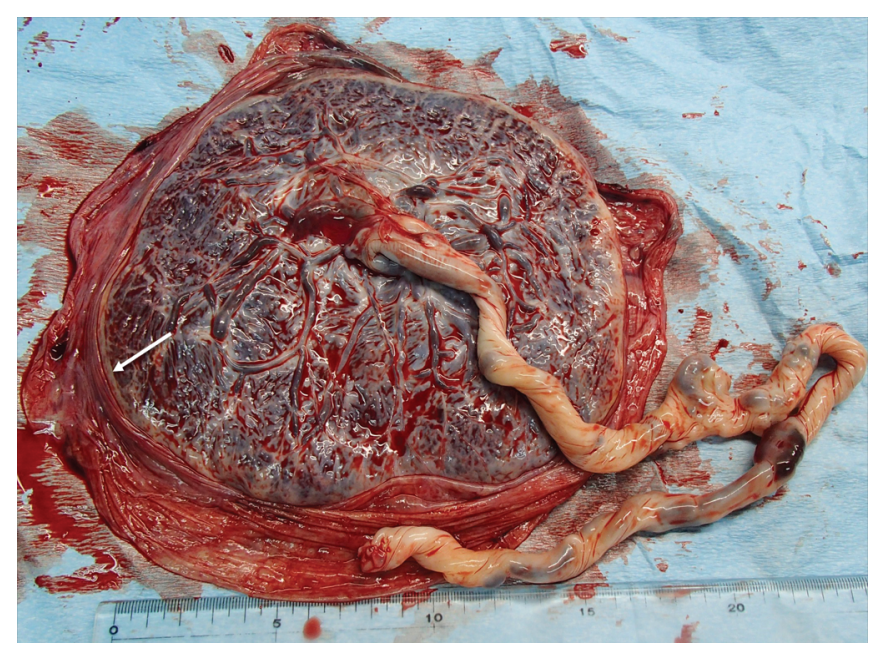

Fig. 6: Gross examination of a partial circumvallate placenta (arrow) after delivery

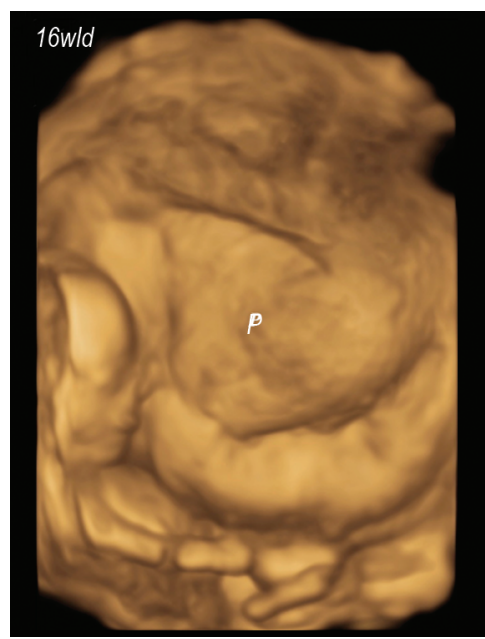

Fig. 8: A circumvallate placenta at 16 weeks and 1 day of gestation. Three-dimensional ultrasound shows a central placental depression with everted placental edges and thick, rolled up placental margins resembling pizza edges surrounding the depressed placental center can be easily depicted. P, placenta

\section{Discussion}

Two-dimensional sonographic diagnosis of a circumvallate placenta depends upon identifying projecting edges of placental margins representing the redundant membranes. ${ }^{8}$ Placental thickness measurement was suggested during 18-21 weeks of gestation to improve the diagnostic accuracy of $2 \mathrm{D}$ sonography in detecting a circumvallate placenta. ${ }^{9}$ In our cases, these characteristic 2D sonographic findings were obtained, and a thick placenta was evident. Moreover, unique 3D ultrasound features were a pizza-like appearance in the second trimester of pregnancy. In previous studies, a characteristic 3D ultrasound finding of a circumvallate placenta in the third trimester was the appearance of a "tire mounted on a wheel" ("tire" sign). ${ }^{4,6}$ The difference in the appearance of the circumvallate placenta between early second and third trimesters is currently unknown. One possible difference is the placental thickness. AboEllail et al. ${ }^{5}$ reported an increased placental

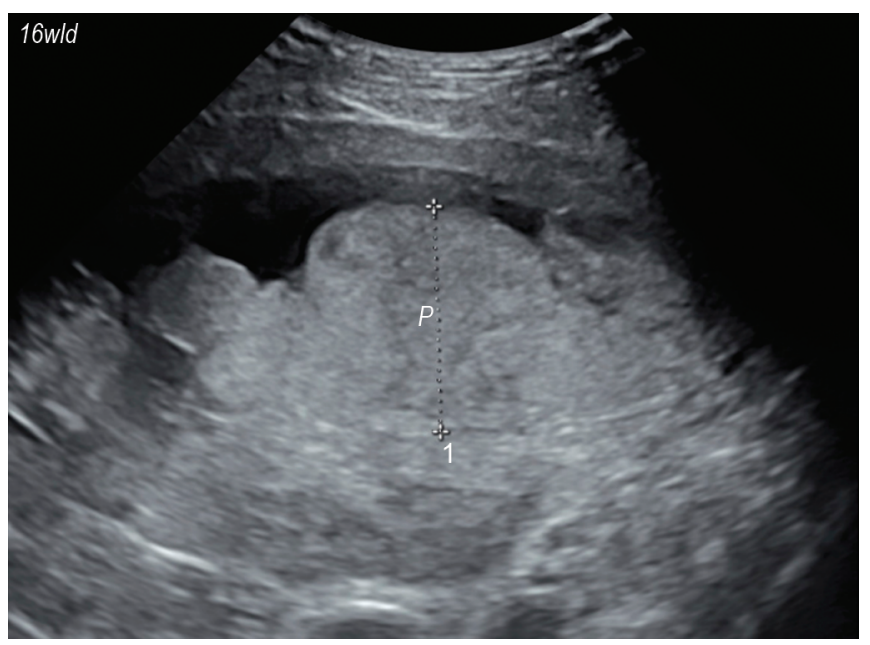

Fig. 7: A circumvallate placenta at 16 weeks and 1 day of gestation. Two-dimensional sonography shows placental edges protruding into the uterine cavity. Placental thickness is $36.5 \mathrm{~mm}$. P, placenta

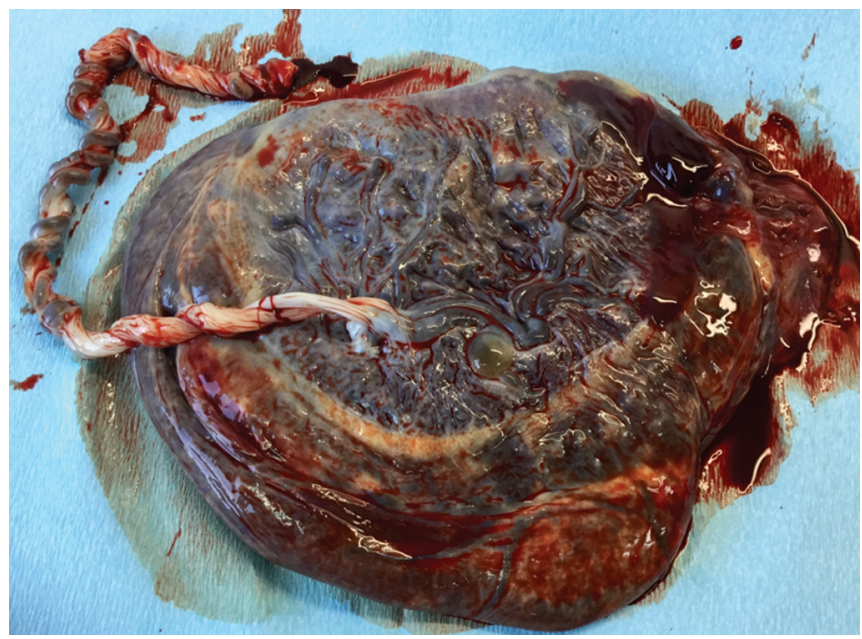

Fig. 9: Gross examination of a complete circumvallate placenta after delivery

thickness in the second-trimester circumvallate placenta, and their HDlive image also showed a pizza-like appearance. Therefore, important diagnostic criteria of the circumvallate placenta early in the second trimester of pregnancy may be projecting edges of placental margins using 2D sonography, increased placental thickness, and a pizza-like appearance on 3D ultrasound.

In the present investigation, there were two partial circumvallate placentas and one complete circumvallate placenta. Twodimensional sonography could not differentiate partial from complete circumvallate placentas, whereas 3D ultrasound and HDlive could clearly demonstrate the partial defect of everted placental edges in the case of a complete circumvallate placenta because the whole placenta could be visualized using 3D ultrasound and HDlive early in the second trimester of pregnancy. These results suggest that $3 \mathrm{D}$ ultrasound and HDlive provide additional, useful information for the early second-trimester diagnosis of a circumvallate placenta. 


\section{References}

1. McCarthy J, Thurmond AS, Jones MK, et al. Circumvallate placenta: sonographic diagnosis. J Ultrasound Med 1995;14(1):21-26. DOI: 10.7863/jum.1995.14.1.21.

2. Harris RD, Wells WA, Black WC, et al. Accuracy of prenatal sonography for detecting circumvallate placenta. Am J Roentgenol 1997;168(6):1603-1608. DOI: 10.2214/ajr.168.6. 9168736.

3. Suzuki S. Clinical significance of pregnancies with circumvallate placenta. J Obstet Gynaecol Res 2008;34(1):51-54.

4. Arlicot $C$, Herve $P$, Simon E, et al. Three-dimensional surface rendering of the chorionic placental plate: the "tire" sign for the diagnosis of a circumvallate placenta. J Ultrasound Med 2012;31(2):340-341. DOI: 10.7863/jum.2012.31.2.340.
5. AboEllail MAM, Kanenishi K, Mori N, et al. HDlive imaging of circumvallate placenta. Ultrasound Obstet Gynecol 2015;46(4):513514. DOI: 10.1002/uog.14839.

6. De Castro Rezende G, Araujo Junior E. Prenatal diagnosis of placenta and umbilical cord pathologies by three-dimensional ultrasound: pictorial essay. Med Ultrason 2015;17(4):545-549.

7. Ples L, Sima RM, Moisei C, et al. Abnormal ultrasound appearance of the amniotic membranes - diagnosis and significance: a pictorial essay. Med Ultrason 2017;19(2):211-215. DOI: 10.11152/mu-844.

8. Sistrom CL, Ferguson JE. Abnormal membranes in obstetrical ultrasound: incidence and significance of amniotic sheets and circumvallate placenta. Ultrasound Obstet Gynecol 1993;3(4):249255. DOI: 10.1046/j.1469-0705.1993.03040249.x.

9. Suzuki S. Antenatal screening for circumvallate placenta. J Med Ultrasonics 2008;35(2):71-73. DOI: 10.1007/s10396-007-0168-5. 